\title{
Healthcare Utilization and Costs of Systemic Lupus Erythematosus by Disease Severity in the United States
}

\author{
Irene B. Murimi-Worstell ${ }^{1}$, Dora H. Lin ${ }^{1}$, Hong Kan ${ }^{1}$, Jonothan Tierce ${ }^{1}$, Xia Wang' ${ }^{2}$, Henk Nab ${ }^{3}$, \\ Barnabas Desta ${ }^{2}$, G. Caleb Alexander ${ }^{1}$, and Edward R. Hammond ${ }^{2}$
}

\begin{abstract}
Objective. To quantify healthcare utilization and costs by disease severity for patients with systemic lupus erythematosus (SLE) in the United States.

Methods. We conducted descriptive analyses of Humedica electronic health record (EHR) data from 2011 to 2015 (utilization analysis) and integrated Optum administrative claims/Humedica EHR data from 2012 to 2015 (cost analysis) for patients with SLE. All-cause utilization outcomes examined were hospitalizations, outpatient visits, emergency department (ED) visits, and prescription drug use. Analyses of costs stratified by disease severity were limited to patients enrolled in an Optum-participating health insurance plan for $\geq 1$ year after the earliest observed SLE diagnosis date. Costs were converted to 2016 US dollars (US\$).

Results. Healthcare utilization was evaluated in 17,257 patients with SLE. Averaged over the 2011-2015 study period, $13.7 \%$ of patients had $\geq 1$ hospitalization per year, $25.7 \%$ had $\geq 1$ ED visit, and $94.4 \%$ had $\geq 1$ outpatient visit. Utilization patterns were generally similar across each year studied. Annually, $88.0 \%$ of patients had $\geq 1$ prescription, including $1.3 \%$ who used biologics. Biologic treatment doubled between 2011 (0.7\%) and 2015 (1.4\%). Cost analyses included 397 patients. From 2012 to 2015 , patients with severe SLE had mean annual costs of $\$ 52,951$, compared with $\$ 28,936$ and $\$ 21,052$ for patients with moderate and mild SLE, respectively. Patients with severe SLE had increased costs in all service categories: inpatient, ED, clinic/ office visits, and pharmacy.

Conclusion. Patients from the US with SLE, especially individuals with moderate or severe disease, utilize significant healthcare resources and incur high medical costs.
\end{abstract}

Key Indexing Terms: claims data, healthcare costs, healthcare utilization, systemic lupus erythematosus, United States

Systemic lupus erythematosus (SLE) is a chronic, multiorgan autoimmune disease with prevalence in the United States

This study was supported by AstraZeneca.

${ }^{I}$ I.B. Murimi-Worstell, PhD, D.H. Lin, MHS, H. Kan, PhD, J. Tierce, CPhil, G.C. Alexander, MD, MS, Johns Hopkins Bloomberg School of Public Health, Baltimore, Maryland, USA ${ }^{2} X$. Wang, PhD, B. Desta, MBA, E.R. Hammond, MD, PhD, MPH, AstraZeneca, Gaithersburg, Maryland, USA; ${ }^{3}$ H. Nab, MD, PhD, AstraZeneca, Cambridge, UK.

$H K$ is a shareholder of GlaxoSmithKline and is an employee of Eli Lilly and Company. XW, BD, and ERH are AstraZeneca employees. HN was an employee of AstraZeneca at the time this study was conducted. GCA was past Chair of the U.S. Food and Drug Administration's Peripheral and Central Nervous System Advisory Committee; has served as a paid advisor to IQVIA; is a cofounding principal and equity holder in Monument Analytics, a healthcare consultancy whose clients include the life sciences industry as well as plaintiffs in opioid litigation; and is a member of OptumRx's National Pharmacy and Therapeutics Committee. This arrangement has been reviewed and approved by Johns Hopkins University in accordance with its conflict of interest policies. IBMW, DHL, HK, JT, and GCA are study investigators contracted by AstraZeneca and Johns Hopkins Bloomberg School of Public Health.

Address correspondence to Dr. E.R. Hammond, AstraZeneca, 200 Orchard Ridge Drive, Gaithersburg, MD 20878, USA. Email:edward.hammond@astrazeneca.com.

Full Release Article. For details see Reprints and Permissions at jrheum.org. Accepted for publication June 11, 2020. ranging from 42 to 300 cases per 100,000 persons across studies ${ }^{1}$. SLE symptoms vary in manifestation and severity and include skin rash, fatigue, fever, hair loss, neuropsychological dysfunction, and joint pain ${ }^{2,3}$. Multiorgan involvement and long-term corticosteroid use result in progressive organ damage, a key feature of SLE, and may increase disease burden ${ }^{2,3}$.

SLE disease progression is associated with organ damage that affects several systems, including musculoskeletal, neuropsychiatric, renal, cardiovascular, and integumentary ${ }^{1,2,3,4}$. As a consequence, disease progression leads to more hospitalizations and increased healthcare costs ${ }^{5,6}$. Long-term treatments for SLE, including corticosteroids and immunosuppressive therapies, are also associated with increased risk of adverse events, such as infections and cardiovascular events. Additionally, corticosteroids may increase the risk of osteoporosis and cataracts. These adverse outcomes further affect healthcare utilization and costs for patients with SLE ${ }^{7}$.

Previous studies evaluating healthcare utilization and costs for patients with SLE were primarily performed using claims data prior to $2010^{6,8,9,10}$. In these studies, patients with SLE were identified as having greater healthcare utilization and higher costs than control patients who were matched on demographics and clinical characteristics ${ }^{6,8,9,10}$.

We provide an updated profile of healthcare utilization and costs among patients with SLE in the US. Focusing on a time 
period after the introduction of biologics for SLE, such as belimumab and rituximab (RTX; used off-label), we performed a retrospective analysis of administrative claims integrated with electronic health record (EHR) data. We assessed healthcare utilization from 2011 to 2015 and evaluated healthcare costs from 2012 to 2015. To gain a deeper understanding of the burden of SLE, we also examined how SLE disease severity may contribute to healthcare costs.

\section{MATERIALS AND METHODS}

Study design. In this study, we conducted a retrospective analysis of EHR data (utilization analysis) and integrated claims and EHR data (cost analysis) for 2 cohorts of patients with SLE in the US. We quantified the following: (1) incidence and prevalence of SLE; (2) healthcare resource use patterns of patients with SLE, including hospitalizations/inpatient visits, outpatient visits including emergency department (ED) and office or clinic visits, and prescription drug use; and (3) annual direct medical and pharmacy costs incurred, stratified by SLE disease severity. Institutional review board approval was not required because this study utilized anonymized administrative claims and EHR data.

Data source. We used Optum administrative claims and EHR data from Humedica Inc. The EHR data contain information for 70 million patients, of whom 50 million are associated with integrated delivery networks (IDN), groups that provide a spectrum of healthcare services. These data include laboratory results; vital signs; body measurements; lifestyle observations; biomarkers; inpatient and outpatient treatments, including written prescriptions; inpatient-administered medications; and provider notes. The Optum claims database includes medical and pharmacy claims from approximately 40 million commercially insured patients and 6.1 million Medicare Advantage enrollees. For the purposes of this study, a project-specific data extract was generated. It included all EHR and integrated claims data for patients who had $\geq 1$ SLE-related healthcare encounter, defined by the presence of International Classification of Diseases, Ninth and Tenth Revisions, Clinical Modification (ICD-9-CM and ICD-10-CM) codes 710.0 or M32.X, respectively, in their EHR. Both the EHR and claims datasets use billing nomenclature to code healthcare services provided. Diagnosis status and procedures performed are coded using ICD-9-CM and ICD-10-CM. Medication use was identified by the National Drug Codes and Healthcare Common Procedure Coding System, J codes.

Patient identification. Patients with SLE were identified in EHR data using algorithms that required patients to be associated with an IDN, to mitigate for the effects of unobserved services, and have $\geq 2$ SLE-related encounters $\geq 60$ days apart and $\geq 1$ pharmacy claim for an SLE-related medication; or $\geq 2$ SLE-related encounters $\geq 60$ days apart, of which $\geq 1$ was a visit to a rheumatologist ${ }^{11}$. An SLE-related healthcare encounter was defined by the assignment of an ICD-9-CM code 710.0 or ICD-10-CM code M32.X to the patient's EHR for hospitalization, office or clinic visit, ED visit, or other outpatient service visit. SLE-related medications included 4 drug classes used to manage SLE: antimalarial medications, immunosuppressive agents, systemic corticosteroids, and biologics.

Disease severity (mild, moderate, or severe) was determined for each patient by a healthcare utilization-based algorithm derived from the severity of diagnoses listed on claims, prescription drug claims, and conditions associated with SLE severity available in nonlaboratory claims (e.g., renal impairment was considered moderate and endstage renal disease was considered severe) $)^{9}$. Disease severity was determined for each patient during each year of the study, and the severity assigned to a particular year was the highest severity category attained by the patient during that year (see Supplementary Table 1, available with the online version of this article, for full description of the SLE severity algorithm).

Incidence and prevalence of SLE. We identified 2 retrospective cohorts, incident and prevalent, from the EHR data to determine annual incidence and prevalence of SLE from 2011 to 2015. An incident case of SLE referred to both newly diagnosed patients and patients whose SLE condition was dormant during the preestimation year. To be considered an incident or a newly diagnosed SLE case in a given year, patients had to meet the SLE case definition between January 1 and December 31 of the year of estimation. Incident patients also had to be included in EHR data for $\geq 365$ days (with no SLE-related codes) before their first SLE-related encounter. To be considered a prevalent SLE case, newly diagnosed or patients with existing SLE had to meet the case definition for SLE and have information in the database during the year of estimation. Patients who did not have information in the database for a given year were not counted during that year. The denominator used for determining yearly incidence and prevalence was the number of patients who had $\geq 1$ encounter during the year.

Patterns of healthcare utilization for patients with SLE. Healthcare utilization was determined for the years 2011 to 2015. Patients were followed from their index dates (earliest SLE diagnosis date observed in the database) until the month of their last observed activities in the EHR database or date of death, whichever was first. All study participants were required to be part of the EHR database during the calendar year of estimation to assess annual healthcare utilization patterns and to have $\geq 1$ calendar year of data after the index date.

All-cause healthcare utilization was measured in the following categories: hospitalizations, outpatient visits (ED and office or clinic visits), total drug prescriptions, and SLE-related drug prescriptions [antimalarial medications, immunosuppressive agents (azathioprine, mycophenolate mofetil, cyclosporine, methotrexate, leflunomide, cyclophosphamide, chlorambucil, nitrogen mustard), systemic corticosteroids, and biologics (RTX, belimumab)]. Disease severity was not measured in this cohort because corticosteroid dose could not be determined for all patients.

Direct medical and pharmacy costs associated with SLE. Healthcare cost analyses were limited to data from 2012 to 2015 in a separate cohort. To be included in the cost analysis, patients had to (1) be identified in the EHR database; (2) be enrolled in an Optum-participating health insurance plan with both medical and pharmacy benefits on the index date; and (3) have $\geq 1$ calendar year of data after the index date.

SLE-related outcomes evaluated were annual direct medical costs for patients with $\geq 1$ service-type encounter, stratified by service type (e.g., hospitalizations, ED visits, office/clinic visits, and other patient services); annual prescription drug costs, including both outpatient- and inpatient-administered drugs; and annual direct medical costs associated with SLE. Study outcomes were also stratified by service type and SLE disease severity (mild, moderate, or severe) $)^{9}$. All costs were converted to 2016 US\$ using the Consumer Price Index annual averages for medical care ${ }^{12}$.

Statistical analyses. Patient demographics were characterized using descriptive statistics. Healthcare utilization for 2011 to 2015 was stratified by year and evaluated using descriptive statistics. Healthcare costs for 2012 to 2015 were categorized and reported annually for hospitalizations, outpatient visits, ED visits, clinic or office visits, outpatient services, and medications. Annual costs were also reported by SLE disease severity. All analyses were performed using SAS version 9.4 (SAS: SAS Institute Inc.).

\section{RESULTS}

Healthcare utilization analysis: patient characteristics. A total of 17,257 patients met the SLE case definition within the EHR database and were included in the analysis of healthcare utilization (Figure 1). The majority were female $(89.7 \%, \mathrm{n}=15,482)$ and white $(69.3 \%, \mathrm{n}=11,962)$. Mean $(\mathrm{SD})$ age was $48.4(15.6)$ years. The distribution of medical insurance coverage among the population was as follows: commercial insurance $32.6 \%$ $(\mathrm{n}=5628) ;$ Medicare 16.6\% $(\mathrm{n}=2864)$; Medicaid $5.5 \%$ 


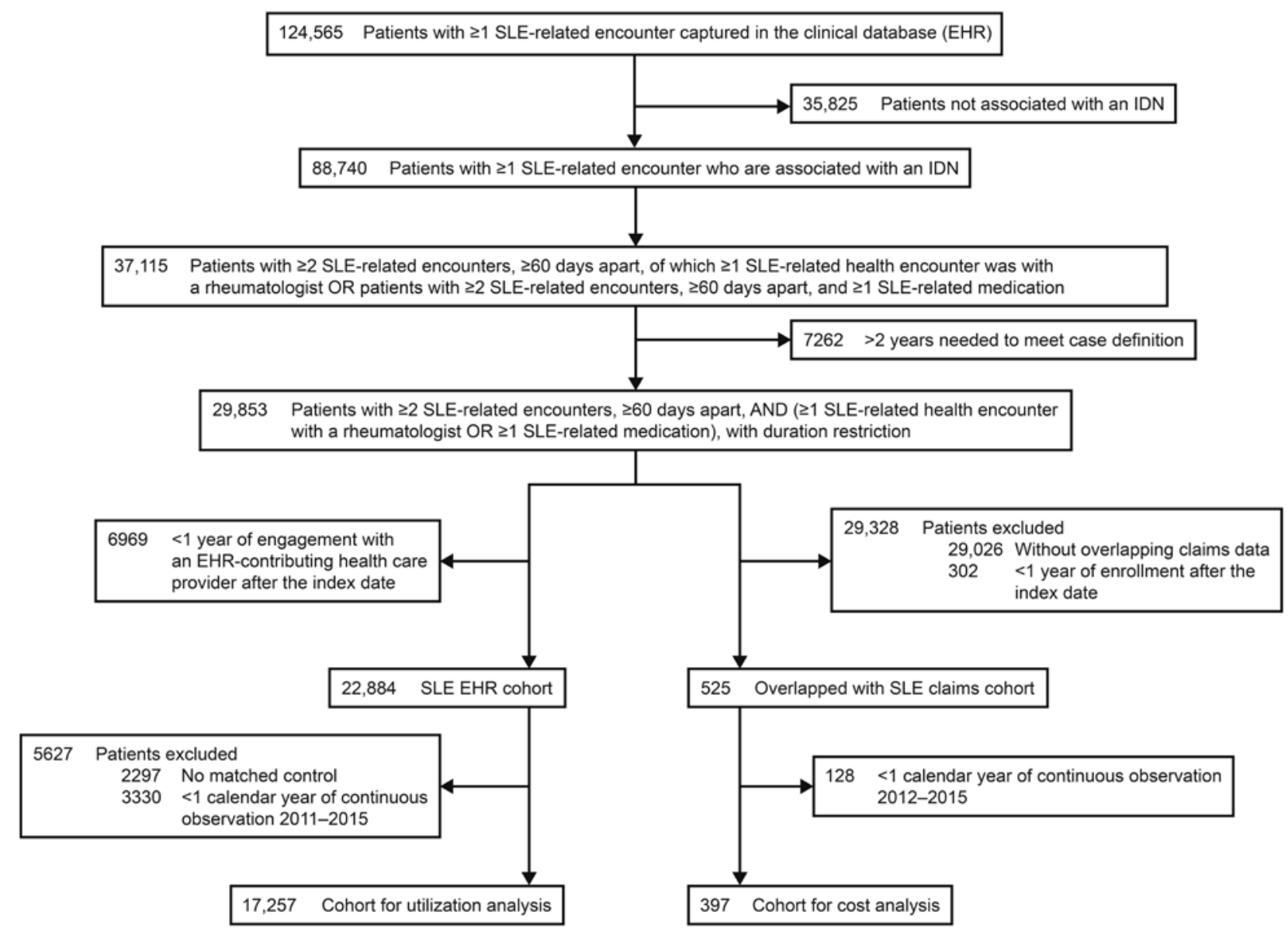

Figure 1. Derivation of the study cohort. EHR: electronic health record; IDN: Integrated Delivery Network; SLE: systemic lupus erythematosus.

$(\mathrm{n}=945)$; unknown insurance coverage $12.8 \%(\mathrm{n}=2215)$; and uninsured 30.4\% ( $\mathrm{n}=5249$; Table 1$)$.

From 2011 to 2015, the yearly incidence of SLE (incident or newly treated SLE cases) ranged from 10.7 to 14.0 per 100,000 patients (Table 2). The estimated prevalence of patients with SLE was 44.1 per 100,000 patients in 2011 , increasing to 85.3 per 100,000 patients in 2015.

Healthcare utilization analysis: all-cause healthcare utilization. Healthcare utilization generally remained the same for each year of the study (2011-2015), except for trends noted in Table 2. For patients with $\geq 1$ outpatient visit, including ED visits, the median (IQR) number of annual visits increased from 15 (7-28) in 2011 to 19 (9-37) in 2015. The proportion of patients with $\geq 1$ ED visit increased from $22.8 \%$ in 2011 to $29.3 \%$ in 2015 . Of patients with $\geq 1$ prescription from 2011 to 2015 , the proportion of those who were prescribed biologics increased from $0.7 \%$ to $1.4 \%$ (Table 2). The proportion of patients prescribed antimalarial medications generally decreased from 2011 to 2015 $(21.2 \%, 19.9 \%, 16.4 \%, 16.8 \%$, and $17.1 \%$, respectively, for each year chronologically). The proportion of patients prescribed systemic corticosteroids decreased each year from 2011 to 2014, with an increase in $2015(21.8 \%, 19.0 \%, 17.7 \%, 17.2 \%$, and $18.3 \%$, respectively, for each year chronologically).
When averaged over the period from 2011 to 2015, 13.7\% of patients had $\geq 1$ hospitalization per year, with a mean (SD) of 1.7 (1.4) hospital visits per year and an average hospital stay of 5.4 (13.0) days (Table 2). Almost all patients had $\geq 1$ outpatient visit (i.e., all noninpatient services, which include ED and clinic or office visits) per year (94.4\%), with a mean (SD) of 24.1 (23.8) visits per year. ED services ( $\geq 1$ visit) were sought by $25.7 \%$ of patients, with a mean (SD) of 2.5 (3.4) visits annually, and $86.3 \%$ of patients had $\geq 1$ clinic or office visit per year, with a mean (SD) of 10.0 (10.2) visits per year. Over the study period from 2011 to 2015 , a mean of $88.0 \%$ of patients had $\geq 1$ prescription claim for any type of medication each year. Systemic corticosteroids (18.8\%), antimalarial medications (18.3\%), immunosuppressants (10.3\%), and biologics (1.3\%) were among the prescribed medications.

Healthcare cost analysis stratified by SLE disease severity: patient characteristics. Data from the 397 patients identified in the EHR database who were also enrolled in an Optum-participating health insurance plan from 2012 to 2015 were utilized in the SLE cost analysis (Figure 1). Baseline characteristics of patients in the cost analysis were similar to those of patients in the healthcare utilization analysis (Table 1). Using the SLE severity algorithm? 256 patients were categorized with mild SLE, 106 with moderate 
Table 1. Characteristics of patients with SLE evaluated in the healthcare utilization cohort, 2011-2015 (Humedica EHR Data), and the healthcare costs cohort, 2012-2015 (Optum/Humedica Integrated Claims/EHR data).

\begin{tabular}{|c|c|c|}
\hline Patient Characteristics & $\begin{array}{l}\text { Healthcare Utilization, } \\
\qquad N=17,257\end{array}$ & $\begin{array}{l}\text { Healthcare Costs } \\
\qquad \mathrm{N}=397\end{array}$ \\
\hline \multicolumn{3}{|l|}{ Age at index date, yrs } \\
\hline Mean $(S D)$ & $48.4(15.6)$ & $51.1(14.9)$ \\
\hline Median (IQR) & $49(37-59)$ & $52(40-62)$ \\
\hline Female, n (\%) & $15,482(89.7)$ & $350(88.2)$ \\
\hline \multicolumn{3}{|l|}{ Race, n (\%) } \\
\hline African American & $3209(18.6)$ & $64(16.1)$ \\
\hline White & $11,962(69.3)$ & $276(69.5)$ \\
\hline Other/unknown & $2086(12.1)$ & $57(14.4)$ \\
\hline \multicolumn{3}{|l|}{ Ethnicity, n (\%) } \\
\hline Hispanic & $1218(7.1)$ & $20(5.0)$ \\
\hline Non-Hispanic & $14,655(84.9)$ & $344(86.6)$ \\
\hline Unknown & $1384(8.0)$ & $33(8.3)$ \\
\hline \multicolumn{3}{|l|}{ Geographic region, n (\%) } \\
\hline Midwest & $7149(41.4)$ & $148(37.3)$ \\
\hline Northeast & $2282(13.2)$ & $118(29.7)$ \\
\hline South & $6056(35.1)$ & $91(22.9)$ \\
\hline West & $1310(7.6)$ & $32(8.1)$ \\
\hline Other/unknown & $460(2.7)$ & $8(2.0)$ \\
\hline \multicolumn{3}{|l|}{ Primary insurance, $\mathrm{n}(\%)^{\mathrm{a}}$} \\
\hline Commercial & $5628(32.6)$ & $272(68.5)$ \\
\hline Medicaid & $945(5.5)$ & 0 \\
\hline Medicare & $2864(16.6)$ & $115(29.0)$ \\
\hline Uninsured & $5249(30.4)$ & 0 \\
\hline Unknown & $2215(12.8)$ & 0 \\
\hline Several & $356(2.1)$ & $10(2.5)$ \\
\hline \multicolumn{3}{|l|}{ Household income, US\$ $\$^{\mathrm{b}}$} \\
\hline Mean (SD) & $41,748(10,206)$ & $43,873(10,948)$ \\
\hline \multicolumn{3}{|l|}{ College educated, $n(\%)^{c}$} \\
\hline$\leq 10 \%$ & $27(0.2)$ & $1(0.3)$ \\
\hline $11-20 \%$ & $5310(30.8)$ & $77(19.4)$ \\
\hline $21-30 \%$ & $8577(49.7)$ & $211(53.1)$ \\
\hline$>30 \%$ & $2882(16.7)$ & $100(25.2)$ \\
\hline Unknown & $461(2.7)$ & $8(2.0)$ \\
\hline
\end{tabular}

${ }^{\mathrm{a}}$ Insurance that was most commonly used in the healthcare utilization cohort for a healthcare encounter. ${ }^{\mathrm{b}}$ Average household income at the individual's 3-digit zip code. 'Percentage college educated by the individual's 3-digit zip code. EHR: electronic health record; SLE: systemic lupus erythematosus.

SLE, and 149 with severe SLE. SLE severity categorization was performed each year throughout the study period. Because SLE severity varies over time, an individual patient may meet $>1$ SLE severity category during the study period. Therefore, the number of patients per severity category represents the time-varying nature of SLE severity and indicates the number of patients who spent time in mild, moderate, and severe SLE states rather than the total number of patients included in the analysis.

Healthcare cost analysis stratified by SLE disease severity: all-cause healthcare utilization in cost cohort. The annual proportion of patients with $\geq 1$ hospitalization or ED visit increased with disease severity (Table 3 ). The proportion of patients hospitalized annually was greater for those with severe SLE (36.9\%) compared with moderate (17.9\%) or mild SLE (16.8\%). ED visits also increased with disease severity; $38.3 \%$ of patients with severe SLE, $31.1 \%$ with moderate SLE, and $28.5 \%$ with mild SLE had $\geq 1$ ED visit each year. Patients prescribed biologics increased with SLE severity over the study period. Patients with mild, moderate, and severe SLE prescribed biologics were $2.0 \%$, $3.8 \%$, and $4.7 \%$, respectively.

Healthcare cost analysis stratified by SLE disease severity: direct medical and pharmacy costs associated with SLE. Over the study period from 2012 to 2015, total mean annual costs for patients with SLE who had $\geq 1$ healthcare encounter were $\$ 32,374$ (Table 3 and Figure 2). Patients with severe SLE had mean annual costs of $\$ 52,951$, compared with $\$ 28,936$ and $\$ 21,052$ for patients with moderate and mild SLE, respectively. The increased costs associated with greater SLE severity were observed for each category of healthcare service: inpatient, ED and clinic/office visits, and pharmacy costs. Total mean annual healthcare costs were 2.5-fold greater for patients with severe SLE compared with patients with mild SLE. Mean hospitalization, ED visit, clinic/ office visit, and pharmacy costs were also greater for patients with severe compared with mild SLE with $\geq 1$ admission/visit/ 


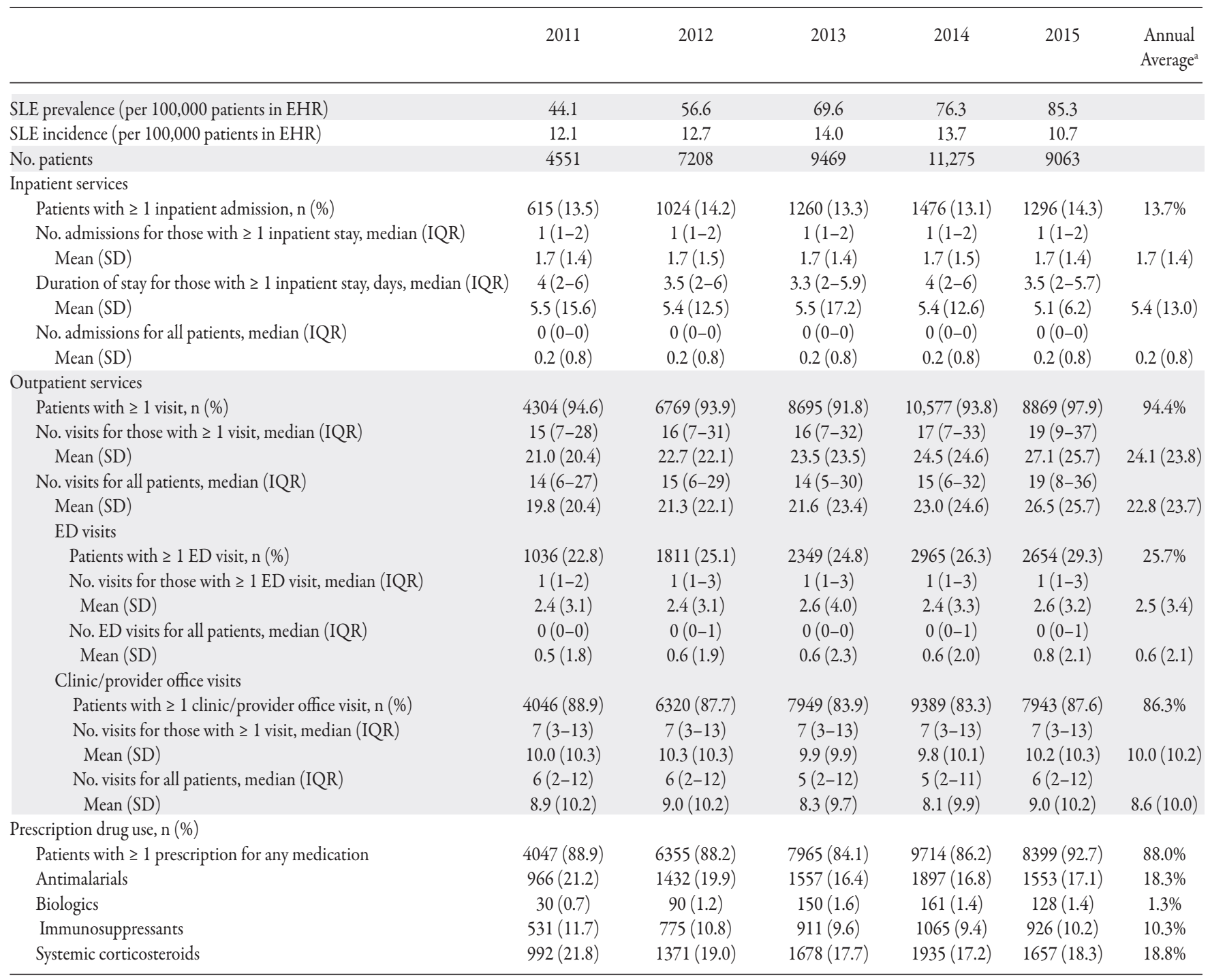

${ }^{a}$ Percentage or mean (SD). As percentages were an annual average of each year of the study, which had different numbers of patients each year, $\mathrm{n}$ values were not given. Total number of patients for the entire study was 17,257. ED: emergency department; EHR: electronic health record; SLE: systemic lupus erythematosus.

prescription (differences were 1.8-, 1.7-, 1.7-, and 2.0-fold, respectively). Total median annual costs analyzed on a yearly basis also increased with SLE severity each year from 2012 to 2015 (Table 4).

\section{DISCUSSION}

In this study, we describe patterns of healthcare utilization and costs among patients with SLE in the US in separate cohorts for utilization and cost analyses. We identified that, overall, patients with SLE utilized significant healthcare resources; between one-fifth and one-third of patients required an ED visit annually, and an average of $13.7 \%$ of patients required inpatient services each year. For patients with SLE, healthcare utilization and costs remained important elements of disease burden from 2011 to 2015 , as was reported prior to $2010^{6,8,9,10}$. Patients with SLE had means of 1.7 hospitalizations, $2.5 \mathrm{ED}$ visits, and 10 clinic/ provider visits annually. By comparison, for the general adult population in the US Healthcare Cost and Utilization Project, hospitalizations ranged from 78.9 to 502.2 per 1000 persons for those 18 to 44 years and $\geq 85$ years of age in $2012^{13}$. In the US National Hospital Ambulatory Medical Care Survey, ED visits for the general adult population ranged from 47 to 61 per 100 persons for those 25 to 44 years and $\geq 75$ years of age in $2015^{14}$.

To the best of our knowledge, this is the first study to combine integrated claims and EHR data to evaluate costs by disease severity for a population with SLE. This approach is advantageous because the data sources complement each other; the EHR database provides a more complete set of patient diagnoses compared with claims records, and claims records provide more complete documentation of services and medications used compared with EHR data. Together, both data sources provide more comprehensive insight into healthcare utilization and costs for patients with SLE than either could provide alone.

Patients with severe disease had markedly greater 
Table 3. Annual healthcare costs associated with SLE, stratified by disease severity and service use, 2012-2015 (Optum/Humedica Integrated Claims/EHR data) ${ }^{a}$.

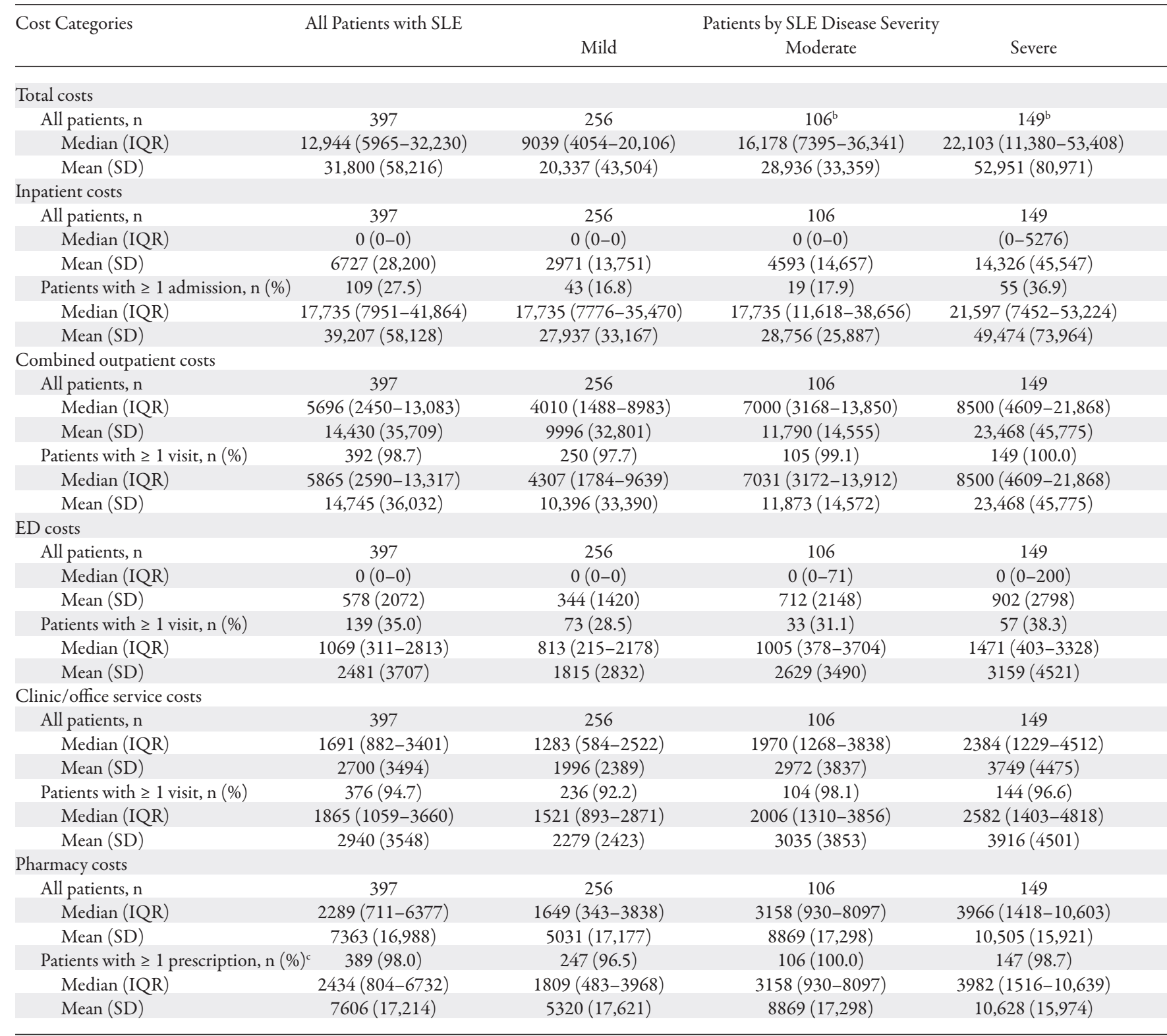

Values are 2016 US\$. ${ }^{a} \mathrm{~N}$ values based on the entire 4-year period; an individual patient may meet $\geq 1$ SLE severity category during the entire study period. Therefore, the number of patients per severity category differs from the total number of patients included in the analysis. ${ }^{\mathrm{b}}$ Antimalarial medications, $\mathrm{n}(\%):$ mild 132 (51.6), moderate 67 (63.2), severe 99 (66.4); biologics, n (\%): mild 5 (2.0), moderate 4 (3.8), severe 7 (4.7); immunosuppressants, n (\%): mild 37 (14.5), moderate 29 (27.4), severe 69 (46.3); systemic corticosteroids, n (\%): mild 64 (25.0), moderate 92 (86.8), severe 105 (70.5). ' By definition, patients with moderate or severe SLE used $\geq 1$ healthcare service during the year. ED: emergency department; EHR: electronic health record; SLE: systemic lupus erythematosus.

healthcare utilization and costs compared with those with mild and moderate disease. This could be potentially because patients with severe disease have previously been shown to have greater organ involvement, faster disease progression, and increased flare frequency and intensity than patients with mild or moderate disease ${ }^{1,2,3,4}$. For patients with severe SLE, the annual rate of hospitalization was 2-fold greater than that of patients with milder forms of SLE. Patients with severe SLE also required $10 \%$ more ED visits than patients with mild disease. The greatest effect of SLE severity on cost was for outpatient visits. Mean annual SLE-related costs for hospitalizations were $\$ 27,937$ for mild SLE compared with $\$ 49,474$ for severe SLE in patients with $\geq 1$ admission; mean annual outpatient visit costs were $\$ 10,396$ for mild SLE compared with $\$ 23,468$ for severe SLE in patients with $\geq 1$ visit.

The relationship between SLE severity and cost was observed during every year in the study and reflects an increased burden for patients with SLE as their disease progresses. Disease progression is associated with multiorgan involvement, including the central nervous, cardiovascular, urinary, and coagulation systems ${ }^{1,2,3,4}$. 


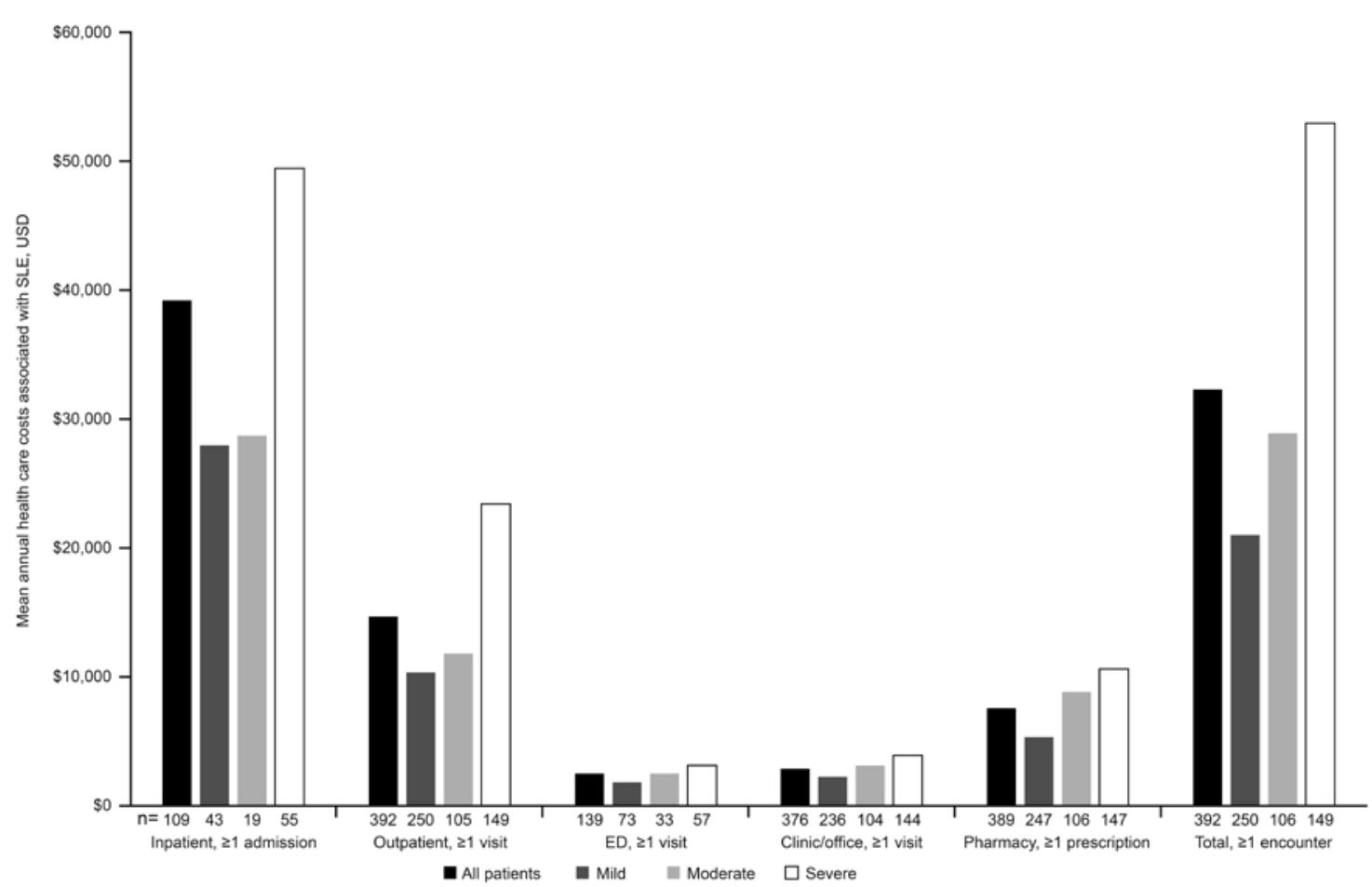

Figure 2. Disease severity and mean annual healthcare costs associated with SLE, 2012-2015 (Optum/Humedica integrated claims/ EHR data). ED: emergency department; EHR: electronic health record; SLE: systemic lupus erythematosus; USD: US dollars.

As disease severity increases, additional treatment is required, as reflected in the mean annual prescription costs for patients with SLE who had $\geq 1$ prescription, which were much greater for severe SLE $(\$ 10,628)$ than mild SLE $(\$ 5320)$. A similar pattern of increasing medical costs with progression to greater disease severity has been reported in a study of 2-year costs of SLE, in which medical costs increased from $\$ 15,117$ to $\$ 61,455$ for patients with moderate versus severe SLE'. Greater disease severity in related disorders, such as rheumatoid arthritis, has also been associated with greater medical costs ${ }^{15}$, and spending $\geq 50 \%$ of the time in a low disease activity state has been associated with a $25.9 \%$ reduction in annual direct medical costs for patients with SLE ${ }^{16}$.

Over the study period from 2012 to 2015, annual total healthcare costs generally remained the same. For the individual cost components, such as inpatient costs, there was some variability that may have been associated with the low number of patients in each group. Median annual pharmacy costs, however, were generally greater every year compared with 2012 , with a $38 \%$ (\$773) increase in 2015. Increases in pharmacy costs over the years may reflect the increased use of biologics.

Corticosteroids were among the most widely used SLE-related medications in our utilization analysis (18.8\%), reflecting a common treatment strategy for management of SLE, flares in particular, in hospital and ED settings ${ }^{17}$. Because corticosteroid use is associated with increased risk of adverse effects ${ }^{7}$, it may increase healthcare utilization and costs in the longer term for patients with SLE. The proportion of patients prescribed antimalarials was between $21.2 \%$ and $17.1 \%$ during the study period, which was somewhat surprising. Use of antimalarials in the treatment of SLE has been shown to be safe and efficacious ${ }^{18}$ and is recommended as per treatment guidelines ${ }^{17}$. Biologic (RTX and belimumab) use was low (1.3\%) and ranged from $0.7 \%$ in 2011 to $1.4 \%$ in 2015 . This finding is similar to that of another US cohort of patients with SLE identified in 2013 to 2015 , in which biologic use at 1 year after diagnosis was $1 \%$ for belimumab and $0.9 \%$ for RTX ${ }^{19}$.

Prevalence rates in the EHR study population increased between 2011 and 2015, with 44.1 per 100,000 patients in 2011, increasing to 85.3 per 100,000 patients in 2015 . This estimated prevalence falls within range of reported SLE prevalence estimates in the US $(20-150 \text { cases per } 100,000)^{20,21,22}$.

There were some limitations to this study. Healthcare utilization patterns were estimated using an open cohort population. Services received outside the EHR reporting network were not available for analyses. To mitigate for the effects of unobserved services, the study was restricted to patients who were associated with an IDN, a group that provides a spectrum of services (e.g., hospital, dispensing pharmacy, outpatient, community care sites) under an umbrella organizational structure. In addition, patients who paid cash for medicines were not captured in the healthcare utilization analysis.

In this study, cost estimates were based on modeled charges rather than actual expenditures, which may result in inaccurately reflected costs for some claims that were not reimbursed. SLE disease severity was determined by a proxy method that has been used previously and validated to identify severe, moderate, and mild SLE9. However, SLE severity may have been misclassified in some patients using this claims-based method. The strengths of this study include the large cohort of patients evaluated and the 
Table 4. Annual healthcare costs associated with SLE, stratified by disease severity, service use, and year (patients with $\geq 1$ SLE-related encounter), 2012-2015 (Optum/Humedica Integrated Claims/EHR data) ${ }^{a}$.

\begin{tabular}{|c|c|c|c|c|}
\hline \multirow[t]{2}{*}{ Cost Categories } & \multirow{2}{*}{ All Patients with SLE } & \multicolumn{3}{|c|}{ Patients by SLE Disease Severity } \\
\hline & & Mild & Moderate & Severe \\
\hline \multicolumn{5}{|l|}{ Total costs } \\
\hline 2012 & $13,116(4649-28,824), 139$ & $8887(3711-15,465), 70$ & $15,584(7354-41,438), 27^{\mathrm{b}}$ & $23,551(9928-50,868), 42^{\mathrm{b}}$ \\
\hline 2013 & $14,346(6557-33,034), 206$ & $10,870(4562-21,897), 108$ & $14,813(9239-38,175), 34^{\mathrm{b}}$ & $19,254(10,892-63,222), 64^{b}$ \\
\hline 2014 & $12,338(5153-32,735), 225$ & 8426 (4135-19,137), 122 & $18,222(8284-38,310), 39^{\mathrm{b}}$ & $23,082(8348-64,752), 64^{\mathrm{b}}$ \\
\hline 2015 & $14,643(6676-34,009), 275$ & $9216(5044-23,710), 142$ & $16,411(6475-27,232), 44^{\mathrm{b}}$ & $22,104(12,429-48,660), 89^{b}$ \\
\hline \multicolumn{5}{|l|}{ Inpatient costs } \\
\hline 2012 & $16,424(10,080-32,331), 21$ & $17,619(10,667-26,020), 6$ & $37,262(16,424-71,253), 3$ & $13,394(9094-26,964), 12$ \\
\hline 2013 & $20,326(7776-46,050), 39$ & $15,147(6847-17,735), 13$ & 17,735 (9893-41,999), 9 & $37,350(20,326-90,972), 17$ \\
\hline 2014 & $28,869(16,213-45,869), 35$ & $17,735(6066-34,783), 11$ & $21,292(17,735-38,656), 5$ & $33,028(24,720-74,366), 19$ \\
\hline 2015 & $16,790(7190-36,722), 50$ & $25,278(14,653-56,863), 17$ & $14,857(11,749-17,735), 6$ & $12,082(6148-36,722), 27$ \\
\hline \multicolumn{5}{|c|}{ Combined outpatient costs } \\
\hline 2012 & $5594(2082-12,382), 135$ & $4153(1768-8983), 67$ & $8280(2822-16,593), 26$ & $8892(4003-17,069), 42$ \\
\hline 2013 & $6661(3080-14,008), 200$ & $5389(2068-12,244), 102$ & $6461(3162-13,912), 34$ & $8475(4680-20,996), 64$ \\
\hline 2014 & 4901 (2516-12,471), 222 & 3720 (1664-7791), 119 & $7032(3834-16,337), 39$ & $10,941(3890-28,772), 64$ \\
\hline 2015 & $6042(2514-14,026), 270$ & $4110(1925-10,066), 137$ & $6717(3014-12,678), 44$ & $8488(5135-21,868), 89$ \\
\hline \multicolumn{5}{|l|}{ ED visit costs } \\
\hline 2012 & $693(298-2155), 26$ & $508(202-874), 9$ & $1005(570-10,894), 7$ & 591 (310-1987), 10 \\
\hline 2013 & $1092(345-3272), 50$ & 557 (238-2012), 19 & $1974(427-4082), 9$ & $1316(363-3879), 22$ \\
\hline 2014 & 1350 (478-2228), 55 & $1722(544-3227), 28$ & 978 (165-2228), 10 & $1272(597-1798), 17$ \\
\hline 2015 & $1023(256-2898), 66$ & 473 (178-1892), 28 & 669 (391-2417), 13 & $2513(617-4372), 25$ \\
\hline \multicolumn{5}{|c|}{ Clinic/office visit costs } \\
\hline 2012 & 1695 (946-3078), 126 & 1465 (843-2699), 61 & 1826 (1085-2739), 26 & 2163 (1190-4272), 39 \\
\hline 2013 & 2242 (1248-3922), 184 & 2054 (977-3049), 92 & $2296(1785-3906), 33$ & 2722 (1554-5565), 59 \\
\hline 2014 & $1718(985-3712), 208$ & 1383 (857-2841), 108 & $2378(1373-5070), 38$ & 2159 (1009-4467), 62 \\
\hline 2015 & 1745 (1109-3577), 258 & 1460 (913-2639), 126 & $1712(1109-2765), 44$ & 2913 (1601-4761), 88 \\
\hline \multicolumn{5}{|l|}{ Pharmacy costs } \\
\hline 2012 & 2043 (712-6197), 134 & $1577(651-3560), 66$ & 3305 (472-9629), 27 & $2901(844-11,495), 41$ \\
\hline 2013 & 2358 (865-6583), 199 & 1626 (577-3899), 101 & $3122(710-7253), 34$ & $3910(1404-10,075), 64$ \\
\hline 2014 & $2279(711-6186), 218$ & 1806 (326-4029), 115 & $3560(808-7506), 39$ & 3580 (1312-9585), 64 \\
\hline 2015 & 2816 (976-7849), 267 & 2159 (569-4139), 136 & $2696(1078-9005), 44$ & $4568(2182-12,295), 87$ \\
\hline
\end{tabular}

Values are median (IQR) in 2016 US\$, n. ${ }^{2}$ An individual patient may meet $\geq 1$ SLE severity category during the entire study period. Therefore, the number of patients per severity category differs from the total number of patients included in the analysis. ${ }^{b}$ By definition, patients with moderate or severe SLE had $\geq 1$ healthcare encounter during the year. ED: emergency department; EHR: electronic health record; SLE: systemic lupus erythematosus.

extension of the time frames that were evaluated in previous studies. In addition, the use of administrative claims integrated with EHR is a unique aspect of this study that allows comprehensive evaluation of healthcare utilization and costs, including analysis of the association between disease severity and costs.

This study provides an updated estimate of the economic burden of SLE and demonstrates that moderate and severe SLE remain a significant driver of healthcare utilization and costs in the US. These findings underscore the importance of developing innovative treatments for SLE that may be both disease-modifying and steroid-sparing to reduce healthcare utilization and costs.

\section{ACKNOWLEDGMENT}

Editorial assistance was provided by Alan Saltzman, PhD, CMPP, and Ellen Stoltzfus, PhD, of JK Associates Inc., a member of the Fishawack Group of Companies, Conshohocken, Pennsylvania, USA.

\section{ONLINE SUPPLEMENT}

Supplementary material accompanies the online version of this article.

\section{REFERENCES}

1. Carter EE, Barr SG, Clarke AE. The global burden of SLE: prevalence, health disparities and socioeconomic impact. Nat Rev Rheumatol 2016;12:605-20.

2. Cojocaru M, Cojocaru IM, Silosi I, Vrabie CD. Manifestations of systemic lupus erythematosus. Maedica 2011;6:330-6.

3. Taraborelli M, Cavazzana I, Martinazzi N, Lazzaroni MG, Fredi M, Andreoli L, et al. Organ damage accrual and distribution in systemic lupus erythematosus patients followed-up for more than 10 years. Lupus 2017;26:1197-204.

4. Lam NC, Ghetu MV, Bieniek ML. Systemic lupus erythematosus: primary care approach to diagnosis and management. Am Fam Physician 2016;94:284-94.

5. Bertsias GK, Salmon JE, Boumpas DT. Therapeutic opportunities in systemic lupus erythematosus: state of the art and prospects for the new decade. Ann Rheum Dis 2010;69:1603-11. 
6. Kan HJ, Song X, Johnson BH, Bechtel B, O'Sullivan D, Molta CT. Healthcare utilization and costs of systemic lupus erythematosus in Medicaid. Biomed Res Int 2013;2013:808391.

7. Bakshi J, Segura BT, Wincup C, Rahman A. Unmet needs in the pathogenesis and treatment of systemic lupus erythematosus. Clin Rev Allergy Immunol 2018;55:352-67.

8. Garris C, Shah M, Farrelly E. The prevalence and burden of systemic lupus erythematosus in a Medicare population: retrospective analysis of Medicare claims. Cost Eff Resour Alloc 2015;13:9.

9. Garris C, Jhingran P, Bass D, Engel-Nitz NM, Riedel A, Dennis G. Healthcare utilization and cost of systemic lupus erythematosus in a US managed care health plan. J Med Econ 2013;16:667-77.

10. Furst DE, Clarke A, Fernandes AW, Bancroft T, Gajria K, Greth W, et al. Resource utilization and direct medical costs in adult systemic lupus erythematosus patients from a commercially insured population. Lupus 2013;22:268-78.

11. Hammond E, Trenz H, Wang X, Tummala R, Desta B, Halpern R. Validation of systemic lupus erythematosus (SLE) diagnosis in claims data using electronic health records (EHR) [abstract]. Pharmacoepidemiol Drug Saf 2017;26:445-6.

12. US Department of Labor, Bureau of Labor Statistics. Consumer Price Index detailed report tables. 2004-2016. [Internet. Accessed October 13, 2020.] Available from: www.bls.gov/bls/news-release/ cpi.htm

13. Weiss AJ, Elixhauser A. Overview of hospital stays in the United States, 2012. Agency for Healthcare Research and Quality. [Internet. Accessed October 13, 2020.] Available from: www.hcup-us.ahrq. gov/reports/statbriefs/sb180-Hospitalizations-United-States-2012. pdf

14. Centers for Disease Control and Prevention. National hospital ambulatory medical care survey: 2015 emergency department summary tables [Internet. Accessed October 13, 2020.] Available from: www.cdc.gov/nchs/data/nhamcs/web_tables/2015_ed_web_ tables.pdf
15. Shafrin J, Tebeka MG, Price K, Patel C, Michaud K. The economic burden of ACPA-positive status among patients with rheumatoid arthritis. J Manag Care Spec Pharm 2018;24:4-11.

16 Yeo AL, Koelmeyer R, Kandane-Rathnayake R, Golder V, Hoi A, Huq M, et al. Lupus low disease activity state is associated with reduced direct healthcare costs in patients with systemic lupus erythematosus. Arthritis Care Res 2019 Jul 8 (E-pub ahead of print).

17. Gordon C, Amissah-Arthur M-B, Gayed M, Brown S, Bruce IN, D'Cruz D, et al. The British Society for Rheumatology guideline for the management of systemic lupus erythematosus in adults. Rheumatology 2018;57:e1-45.

18. Ruiz-Irastorza G, Ramos-Casals M, Brito-Zeron P, Khamashta MA. Clinical efficacy and side effects of antimalarials in systemic lupus erythematosus: a systematic review. Ann Rheum Dis 2010;69:20-8.

19. Kariburyo F, Xie L, Sah J, Li N, Lofland JH. Real-world medication use and economic outcomes in incident systemic lupus erythematosus patients in the United States. J Med Econ 2020; 23:1-9.

20. Pons-Estel GJ, Alarcón GS, Scofield L, Reinlib L, Cooper GS. Understanding the epidemiology and progression of systemic lupus erythematosus. Semin Arthritis Rheum 2010;39:257-68.

21. Lawrence RC, Helmick CG, Arnett FC, Deyo RA, Felson DT, Giannini EH, et al. Estimates of the prevalence of arthritis and selected musculoskeletal disorders in the United States. Arthritis Rheum 1998;41:778-99.

22. Chakravarty EF, Bush TM, Manzi S, Clarke AE, Ward MM. Prevalence of adult systemic lupus erythematosus in California and Pennsylvania in 2000: estimates obtained using hospitalization data. Arthritis Rheum 2007;56:2092-4. 